\title{
Prevalência de transtornos alimentares em trabalhadores urbanos de município do Nordeste do Brasil
}

\author{
Prevalence of eating disorders in urban workers \\ in a city of the northeast of Brazil
}

\author{
Ana Paula Kalil Prisco ${ }^{1}$ \\ Tânia Maria de Araújo ${ }^{1}$ \\ Maura Maria Guimarães de Almeida ${ }^{1}$ \\ Kionna Oliveira Bernardes Santos ${ }^{1}$
}

${ }^{1}$ Departamento de Saúde, Universidade Estadual de Feira de Santana. Km 3 BR 116 Campus Universitário VI Módulo, Campus Universitário. 44031-460 Feira de Santana BA. anapaula_kalil@hotmail.com

\begin{abstract}
This article seeks to estimate the prevalence of eating disorders among workers living in urban areas of Feira de Santana, Bahia. It involves an epidemiologic, descriptive and exploratory cross-sectional study of 1,273 randomly selected workers. Socio-demographic and work characteristics, lifestyle, psychosocial aspects at work and eating behavior were investigated. Females were predominant (60.2\%), the youngest $51.7 \%$ were up to 35 years old, married (53.1\%), AfroBrazilian descent (55.2\%), with high school education (51.5\%) and with low incomes (81.2\%). The prevalence of Binge Eating Disorder and Bulimia Nervosa were, respectively, $4.3 \%$ and $1 \%$. The prevalence of these disorders was higher among workers who indulged in excessive alcohol consumption, were dissatisfied with their personal appearance and weight, reported violence in childhood and had financial problems or related worries. Also included were those involved in house care services and commerce, who had informal work arrangements or were in highly demanding work environments. Besides investigating the relation between eating disorders and work, data was provided about these disorders in a population-based study. It is hoped that this study may encourage investments in health policies for these disorders.
\end{abstract}

Key words Eating disorders, Bulimia, Binge eating, Workers
Resumo Este artigo tem por objetivo, estimar a prevalência de transtornos alimentares em trabalhadores residentes em área urbana de Feira de Santana, Bahia. Trata-se de estudo epidemiológico de corte transversal exploratório, incluindo 1.273 trabalhadores, aleatoriamente selecionados. Características sociodemográficas e do trabalho, hábitos de vida, aspectos psicossociais do trabalho e transtornos alimentares foram investigados. Foi predominante o sexo feminino $(60,2 \%)$, os mais jovens (51,7\% tinham até 35 anos), casados (53,1\%), de cor parda (55,2\%), com ensino médio $(51,5 \%)$ e com baixa renda $(81,2 \%)$. As prevalências de Transtorno da Compulsão Alimentar Periódica e Bulimia Nervosa foram, respectivamente, 4,3\% e 1,0\%. As prevalências foram mais elevadas entre os que apresentaram consumo abusivo de álcool, insatisfação com o peso, história de agressão na infância, problema financeiro/preocupações, pertenciam aos setores de serviços domésticos e comércio, os que tinham vinculo informal de trabalho e os que estavam no grupo de trabalho de alta exigência. Além de investigar a relação entre os transtornos alimentares e o trabalho, obteve-se dados sobre esses transtornos em estudo de base populacional. Espera-se que os achados obtidos possam fomentar politicas de saúde para os transtornos alimentares.

Palavras-chave Transtornos alimentares, Bulimia, Compulsão Alimentar, Trabalhadores 


\section{Introdução}

Estudos têm apontado aumento da incidência dos transtornos alimentares (TA $)^{1}$. Entretanto, não há consenso se essa incidência está realmente em ascensão ou não ${ }^{2}$. O aumento pode estar relacionado ao maior conhecimento e divulgação do assunto, levando ao diagnóstico mais preciso dos casos ${ }^{3}$. Por outro lado, refere-se à possibilidade de subestimação das taxas, especialmente em estudos de amostras clínicas, pois geralmente os casos mais graves da doença é que buscam algum tipo de serviço especializado ${ }^{2}$.

Os TA são patologias caracterizadas por alterações no comportamento alimentar. Ocorrem mais frequentemente no sexo feminino, representando $90 \%$ dos casos ${ }^{4}$. A prevalência dessas patologias é baixa, variando de 0,5 a $4,2 \%{ }^{1,5}$. Entretanto, são apontados como um dos transtornos mais comuns entre mulheres jovens e com elevados índices de morbidade e mortalidade ${ }^{1}$. Os TA mais comuns são: anorexia nervosa (AN), bulimia nervosa $(\mathrm{BN})$ e transtorno da compulsão alimentar periódica (TCAP).

A etiologia dos TA é multifatorial, sendo pertinente investigar sua possível relação com o trabalho, uma vez que a inserção no mercado de trabalho pode favorecer a saúde ou a doença, a depender do papel que a atividade laboral tenha na vida do sujeito e das condições nas quais o trabalho é realizado. O trabalho pode ser fonte de prazer e realização, mas também de conflito, estresse e insatisfação ${ }^{6}$.

Existe carência de informações sobre o nexo entre TA e aspectos do trabalho. Refere-se maior ocorrência desses transtornos entre modelos, atores, bailarinos, atletas e jóqueis, para os quais a imagem/peso corporal influencia o sucesso profissional ${ }^{7}$. É possível que outros fatores como estresse contínuo, pressão social em relação ao desempenho e à rotina de trabalho sejam elementos que tornem estes e outros profissionais mais vulneráveis ao desenvolvimento de TA.

Foi adotado, dentre os modelos de estudo que visam contribuir para o entendimento do processo saúde-doença do trabalhador, o Modelo Demanda-Controle, proposto por Karasek ${ }^{8}$, para análise das relações entre estresse ocupacional e repercussões sobre o comportamento alimentar, uma vez que o estresse pode levar ao aumento das compulsões alimentares ${ }^{9}$.

Neste estudo, pretende-se descrever a prevalência de comportamentos sugestivos de BN e TCAP em trabalhadores, segundo características sociodemográficas, comportamental e do tra- balho profissional, com destaque para variáveis relacionadas ao estresse ocupacional.

Trata-se de um estudo pioneiro no município de Feira de Santana envolvendo trabalhadores e TA, a partir do qual se poderá fornecer subsídios para se repensar as ações e as políticas voltadas à saúde do trabalhador, além da ampliação do conhecimento sobre os TA com base em estudos populacionais.

\section{Materiais e métodos}

Realizou-se estudo epidemiológico de corte transversal de caráter exploratório na zona urbana do município de Feira de Santana, Bahia. Trata-se de uma cidade, situada no recôncavo baiano, localizada num entroncamento rodoviário de grande circulação e tendo no comércio e na pecuária os dois pontos fortes de sua economia. Encontra-se à $108 \mathrm{Km}$ da capital Salvador, possui 571.997 habitantes na sua maioria jovem (cerca de $53 \%$ são mulheres $)^{10}$.

Este estudo é um recorte de uma ampla investigação sobre a saúde mental conduzida em amostra representativa da população da zona urbana de Feira de Santana, com 15 anos ou mais de idade. Para o cálculo do tamanho da amostra se assumiu prevalência estimada de transtornos mentais comuns de $25 \%{ }^{11}$, erro amostral de $3 \%$, com 95\% de confiança. Para ajustar o efeito do desenho de estudo, dobrou-se o tamanho da amostra; considerando-se as recusas e as perdas acrescentou-se $20 \%$.

A seleção das áreas foi feita por amostragem estratificada por subdistritos, adotando-se procedimento aleatório, a partir de dados censitários do Instituto Brasileiro de Geografia e Estatística (IBGE), tendo o domicílio como unidade amostral. A amostragem foi feita por etapas múltiplas sucessivas.

Neste estudo, apenas a população trabalhadora foi incluída na análise. Foram considerados elegíveis todos os trabalhadores com 15 anos de idade ou mais que desenvolviam atividade laboral remunerada com ou sem vínculo empregatício na época da entrevista.

\section{Instrumentos de coleta de dados}

Realizaram-se entrevistas com cada trabalhador, utilizando dois instrumentos: ficha domiciliar e questionário individual - todos preenchidos por entrevistador. A ficha domiciliar incluiu informações sobre o domicílio (endereço, número 
de cômodos, infraestrutura existente) e os seus residentes (lista de todos os moradores, identificando sexo e idade). O questionário individual incluiu blocos de questões sobre características sociodemográficas, do comportamento alimentar e trabalho profissional, aspectos psicossociais do trabalho, uso de bebidas alcoólicas, hábito de fumar, atividades de lazer, insatisfação com o peso/ aparência, obesidade autorreferida, agressão na infância e problemas financeiros/preocupações.

Para avaliar o comportamento alimentar, utilizou-se o Patient Health Questionnaire $(\mathrm{PHQ})^{12,13}$. Os TA foram mensurados por dois grupos de questões (totalizando sete), com respostas dicotômicas (sim/não): Grupo I (questões comuns para TCAP e BN: "Você normalmente sente que não consegue controlar o que você come e quanto come?", "Você frequentemente come, dentro de um período de 2 horas, o que a maioria das pessoas consideraria uma grande quantidade de comida?", "Isso ocorreu, em média, com uma frequência de duas vezes na semana pelos últimos 3 meses?”) e Grupo II (questões específicas para BN: "Forçou o vômito?”, “Tomou mais do que duas vezes a dose recomendada de laxantes?", "Jejuou - não comeu absolutamente nada por pelo menos 24 horas?" "Fez exercícios físicos por mais de uma hora especificadamente para deixar de ganhar peso depois de comer exageradamente?").

Respostas afirmativas para as três questões do Grupo I classificam os casos de TCAP. Para $\mathrm{BN}$, além de responder “sim” para estas três questões, o entrevistado deve responder afirmativamente para, pelo menos, uma questão do Grupo $\mathrm{II}^{12}$. O bloco de questões sobre transtornos alimentares do Patient Health Questionnaire (PHQ) apresentou boa sensibilidade (89\%) e especificidade (94\%) em estudo de validação realizado por Spitzer et al. ${ }^{13}$.

\section{Variáveis descritoras}

Por se tratar de um estudo exploratório, pretendeu-se estimar as prevalências dos TA segundo fatores de exposição referidos na literatura: características sociodemográficas (idade, sexo, situação conjugal, escolaridade, renda, cor da pele autorreferida), hábitos de vida (uso de bebida alcoólica (avaliado pelo CAGE), hábito de fumar e atividade de lazer), insatisfação com o peso/ aparência, obesidade autorreferida, agressão na infância e problemas financeiros ou preocupações, características do trabalho profissional (ocupação, vínculo de trabalho [formal e informal] e carga horária semanal) e características psicossociais do trabalho (avaliadas pelo Job Content Questionnaire [JCQ]).

O JCQ é um questionário estruturado proposto por $\mathrm{Karasek}^{14}$, composto de questões que mensuram: controle sobre o próprio trabalho e demanda psicológica. A partir de combinações entre os níveis de demanda e controle, têm-se quatro grupos de experiência de trabalho: baixa exigência (alto controle e baixa demanda), trabalho passivo (baixo controle e baixa demanda), trabalho ativo (alta demanda e alto controle) e alta exigência (alta demanda e baixo controle). Este último foi considerado o grupo de maior exposição ${ }^{15}$ e o de baixa exigência como o de referência.

\section{Análise dos dados}

Realizou-se a caracterização da população estudada. Posteriormente, estimaram-se as prevalências dos TA em trabalhadores. A análise dos fatores associados aos transtornos foi feita a partir do cálculo das razões de prevalência e respectivos intervalos de confiança a 95\%. Observou-se significância estatística para um $\alpha=0,05 \%(\mathrm{p}<0,05)$.

Esta pesquisa foi aprovada pelo Comitê de Ética em pesquisa com seres humanos da Universidade Federal de Feira de Santana (UEFS).

\section{Resultados}

\section{Características da população de estudo}

Dos 1.273 trabalhadores, $60,2 \%$ eram do sexo feminino. Predominaram os mais jovens (51,7\% tinham até 35 anos), casados $(53,1 \%)$, os que se autorreferiram pardos $(55,2 \%)$, com nível escolar médio (51,5\% tinham ensino médio e apenas $4,5 \%$ nível superior) e baixa renda (81,2\% recebiam até dois salários mínimos).

Predominaram os autônomos $(35,4 \%)$ e assalariados $(34,3 \%)$, os empregados nos setores de serviços em geral $(31,8 \%)$ e comércio $(30,5 \%)$, o vínculo informal de trabalho (71\%) e carga horária semanal superior a 40 horas $(44,5 \%)$, denotando elevada jornada de trabalho (Tabela 1).

Sobre os hábitos de vida, constatou-se que, entre os que apresentaram CAGE positivo $(\mathrm{N}=$ 91, 7,1\% do total), 59,3\% eram do sexo masculino. Entre os fumantes ( $\mathrm{N}=169,13,3 \%$ do total), $53,8 \%$ eram homens. Dos trabalhadores que não realizavam atividade regulares de lazer $(\mathrm{N}=151$, $11,9 \%$ do total), $74,2 \%$ era do sexo feminino. 
Tabela 1. Caracterização dos trabalhadores da zona urbana segundo variáveis do trabalho profissional. Feira de Santana, Bahia, 2007.

\begin{tabular}{lrr}
\hline \multicolumn{1}{c}{ Variáveis (N) } & n & \% \\
\hline Ocupação (1.232) & 447 & 36,3 \\
Autônomo & 422 & 34,3 \\
Assalariado com/sem comissão & 120 & 9,7 \\
Empregado doméstico & 110 & 8,9 \\
Funcionário público & 110 & 8,9 \\
Biscateiro & 14 & 1,1 \\
Empregador/empresário & 9 & 0,7 \\
Profissional liberal & & \\
Setor de trabalho (1.248) & 397 & 31,8 \\
Serviços em geral & 381 & 30,5 \\
Comércio & 176 & 14,1 \\
Serviços domésticos & 109 & 8,7 \\
Indústria manufatureira & 107 & 8,6 \\
Ensino & 43 & 3,4 \\
Construção civil & 35 & 2,8 \\
Transporte & & \\
Vínculo (1.260) & 365 & 29,0 \\
Formal & 895 & 71,0 \\
Informal & & \\
Carga horária semanal (1.255) & 198 & 15,8 \\
Até 20h & 153 & 12,2 \\
21 a 30h & 346 & 27,6 \\
31 a 40h & 558 & 44,5 \\
41h ou mais & & \\
\hline
\end{tabular}

* Inclui atividades de serviços em geral como estética, saúde, segurança, esporte, laboratório.

Entre os trabalhadores estudados, $8,2 \%$ consideravam-se obesos, 35,4\% estavam insatisfeitos com o peso/aparência, $12,7 \%$ referiram ter sofrido agressão na infância e $64,4 \%$ tinham problemas financeiros ou preocupações.

\section{Características ocupacionais}

O sexo feminino se destacou em todas as ocupações, exceto entre os assalariados e empregadores/empresários. Os casados e com renda até dois salários mínimos foram predominantes. Autônomos, biscateiros e empregados domésticos apresentaram os maiores percentuais de renda mensal inferior a um salário mínimo: 40,0\%, $74,5 \%$ e $85,8 \%$, respectivamente. Mais de $50 \%$ dos profissionais liberais, assalariados e empregados domésticos tinham idade máxima de 35 anos. Por outro lado, mais de $60 \%$ dos trabalhadores autônomos, funcionários públicos e empregadores/empresários tinham idade superior a 35 anos. Entre os autônomos, biscateiros e empregados domésticos prevaleceu o ensino fundamental e nas demais ocupações o ensino mé- dio, apontando para o baixo nível de escolaridade entre os trabalhadores no primeiro grupo (Tabela 2).

Entre os entrevistados, $45 \%$ referiram alta demanda psicológica e $50,6 \%$ baixo controle sobre o próprio trabalho. As mulheres apresentaram frequências mais elevadas em todos os grupos do Modelo Demanda-Controle (baixa exigência, trabalho passivo, trabalho ativo e alta exigência), especialmente em relação ao grupo de alta exigência $(63,4 \%)$.

As ocupações com percentuais mais elevados no grupo de alta exigência foram os biscateiros (36,5\%), os empregados domésticos $(31,6 \%)$ e os assalariados $(29,1 \%)$.

Trabalhadores com carga horária semanal e" 40 horas apresentaram discreto aumento no grupo de alta exigência $(26,9 \%)$ quando comparado com os que tinham carga horária $<40$ horas $(22,8 \%)$. O percentual de trabalhadores formais e informais no grupo de alta exigência foi, respectivamente, $24,0 \%$ e $27,0 \%$.

\section{Ocorrência dos transtornos \\ do comportamento alimentar}

A prevalência global de TCAP e $\mathrm{BN}$ foram respectivamente: $4,3 \%$ e $1,0 \%$.

A prevalência de TCAP foi similar entre os sexos. Os mais jovens (até 35 anos de idade) apresentaram prevalência 2,22 vezes maior do que indivíduos acima de 35 anos $(\mathrm{p}=0,005)$. Apresentaram prevalências discretamente maiores os que tinham ensino fundamental/analfabeto $(4,3 \%)$ e pessoas de pele parda/preta $(4,5 \%)$. Tiveram maiores prevalências os solteiros $(5,9 \%)$ e os que recebiam até dois salários mínimos (4,4\%) (Tabela 3).

Não se observou diferença na prevalência de $\mathrm{BN}$ entre os sexos. Apresentaram maiores prevalências desse transtorno os mais jovens $(1,5 \%)$, casados $(1,3 \%)$, que tinham ensino fundamental/analfabeto $(1,3 \%)$, cor branca/outras $(1,5 \%)$, e renda mensal inferior a um salário mínimo $(1,4 \%)$. Contudo, nenhumas das diferenças observadas foram estatisticamente significantes (Tabela 3).

Entre os trabalhadores que apresentaram CAGE positivo, a prevalência de TCAP foi 2,94 vezes maior do que entre aqueles que tinham CAGE negativo $(p=0,001)$. Para BN essa diferença foi ainda mais acentuada: 3,9 vezes maior para os que tiveram CAGE positivo $(\mathrm{p}=0,059)$. A prevalência dos transtornos estudados foi maior entre os não fumantes. Verificou-se maior prevalência de TCAP entre aqueles que reali- 
zavam atividade de lazer e o contrário em relação à BN (Tabela 3).

A prevalência de TCAP foi 4,22 vezes maior entre os que estavam insatisfeitos com o peso/aparência ( $\mathrm{p}<0,001)$ quando comparados com os que estavam satisfeitos. Em relação à BN, essa diferença foi mais elevada: 6,19 vezes maior para os que estavam insatisfeitos $(\mathrm{p}=0,002)$. Entre os que se autorreferiram obesos, a prevalência de TCAP foi de $13,5 \%$ e a de $B N$ foi de $1,9 \%$; sendo 4,49 vezes maior entre os que se autorreferiram obesos em comparação aos que se consideraram com peso normal $(\mathrm{p}<0,001)$. Entre os que afirmaram ter sofrido agressão na infância, a prevalência de TCAP foi 2,79 vezes maior quando comparados com os que não viveram tal situação $(p=0,001)$. Para BN esta diferença foi de 2,07 vezes, porém sem significância estatística. Observou-se que as prevalências de TCAP e BN foram mais elevadas entre os que relataram problemas financeiros/preocupações (4,7\% e 1,4\%, respectivamente) (Tabela 3$)$.

A prevalência de TCAP foi mais elevada entre os que tinham ocupações de biscateiro e empregador/empresário (respectivamente, 5,5\% e
$7,1 \%)$, vínculo informal $(4,3 \%)$ e carga horária de até 30 horas semanais $(5,4 \%)$. Entre os profissionais liberais não houve nenhum caso de TCAP (Tabela 4).

A prevalência de $\mathrm{BN}$ foi mais acentuada entre os biscateiros, autônomos e empregados domésticos (em média 1,8\%), e entre trabalhadores com vínculo de trabalho informal (1,3\%). A prevalência de BN foi 4,12 vezes maior entre os que tinham carga horária semanal de até 30 horas quando comparados com os que a tinham superior a 30 horas $(p=0,007)$. Não se observou casos de $\mathrm{BN}$ entre os profissionais liberais, funcionários públicos e empregadores (Tabela 4).

Quanto aos aspectos psicossociais do trabalho, observou-se maior prevalência de TCAP no grupo de trabalho de alta exigência (5,9\%). A prevalência de TCAP foi 2,10 vezes maior para o grupo de alta exigência quando comparado com o de baixa exigência $(p=0,05)$. A prevalência de $\mathrm{BN}$ também foi mais elevada no grupo de alta exigência $(4,15 \%)$, chegando a ser 4,15 vezes maior em relação ao grupo de trabalho de baixa exigência $(\mathrm{p}=0,10)$ (Tabela 5).

Tabela 2. Distribuição das características sociodemográficas, segundo ocupação profissional entre trabalhadores da zona urbana de Feira de Santana, BA, 2007.

\begin{tabular}{|c|c|c|c|c|c|c|c|c|c|c|c|c|c|c|}
\hline \multirow[t]{2}{*}{ Variáveis (N) } & \multicolumn{2}{|c|}{ Autônomo } & \multicolumn{2}{|c|}{$\begin{array}{c}\text { Profissional } \\
\text { liberal }\end{array}$} & \multicolumn{2}{|c|}{$\begin{array}{l}\text { Assalariado } \\
\text { com ou sem } \\
\text { comissão }\end{array}$} & \multicolumn{2}{|c|}{ Biscateiro } & \multicolumn{2}{|c|}{$\begin{array}{l}\text { Empregado } \\
\text { doméstico }\end{array}$} & \multicolumn{2}{|c|}{$\begin{array}{l}\text { Funcionário } \\
\text { público }\end{array}$} & \multicolumn{2}{|c|}{$\begin{array}{c}\text { Empregador/ } \\
\text { empresário }\end{array}$} \\
\hline & $\mathrm{N}$ & $\%$ & $\mathbf{N}$ & $\%$ & $\mathbf{N}$ & $\%$ & $\mathbf{N}$ & $\%$ & $\mathbf{N}$ & $\%$ & $\mathbf{N}$ & $\%$ & $\mathbf{N}$ & $\%$ \\
\hline \multicolumn{15}{|l|}{ Sexo (1.273) } \\
\hline Masculino & 176 & 39,4 & 4 & 44,4 & 213 & 50,5 & 52 & 47,3 & 1 & 0,8 & 39 & 35,5 & 9 & 64,3 \\
\hline Feminino & 271 & 60,6 & 5 & 55,6 & 209 & 49,5 & 58 & 52,7 & 119 & 99,2 & 71 & 64,5 & 5 & 35,7 \\
\hline \multicolumn{15}{|l|}{ Faixa etária (1.273) } \\
\hline 15 a 25 anos & 59 & 13,2 & 4 & 44,4 & 128 & 30,3 & 29 & 26,4 & 37 & 30,8 & 6 & 5,5 & 1 & 7,1 \\
\hline 26 a 35 anos & 118 & 26,4 & 3 & 33,3 & 157 & 37,2 & 27 & 24,5 & 35 & 29,2 & 23 & 20,9 & 2 & 14,3 \\
\hline 36 a 45 anos & 125 & 28,0 & 2 & 22,2 & 70 & 16,6 & 25 & 22,7 & 27 & 22,5 & 32 & 29,1 & 7 & 50,0 \\
\hline 46 anos ou mais & 145 & 32,4 & - & - & 67 & 15,9 & 29 & 26,4 & 21 & 17,5 & 49 & 44,5 & 4 & 28,6 \\
\hline \multicolumn{15}{|l|}{ Situação conjugal (1.269) } \\
\hline Solteiro & 118 & 26,5 & 5 & 55,6 & 176 & 41,7 & 36 & 33,3 & 60 & 50,0 & 27 & 24,5 & 1 & 7,1 \\
\hline Casado/união livre & 268 & 60,1 & 4 & 44,4 & 206 & 48,8 & 54 & 50,0 & 44 & 36,7 & 70 & 63,6 & 10 & 71,4 \\
\hline Divorc/desq/separ/viúv. & 60 & 13,5 & - & - & 40 & 9,5 & 18 & 16,7 & 16 & 13,3 & 13 & 11,8 & 3 & 21,4 \\
\hline \multicolumn{15}{|l|}{ Escolaridade (1.269) } \\
\hline Analfabeto & 5 & 1,1 & - & - & 3 & 0,7 & 10 & 9,1 & 4 & 3,4 & - & - & 1 & 7,1 \\
\hline Ensino fundamental & 231 & 51,8 & 1 & 11,1 & 120 & 28,6 & 58 & 52,7 & 82 & 68,9 & 24 & 21,8 & 5 & 35,7 \\
\hline Ensino médio & 207 & 46,4 & 4 & 44,4 & 278 & 66,2 & 42 & 38,2 & 32 & 26,9 & 62 & 56,4 & 8 & 57,1 \\
\hline Superior & 3 & 0,7 & 4 & 44,4 & 19 & 4,5 & - & - & 1 & 0,8 & 24 & 21,8 & - & - \\
\hline \multicolumn{15}{|l|}{ Renda mensal* (1.270) } \\
\hline Menos que $1 \mathrm{SM}$ & 179 & 40,0 & 2 & 22,2 & 92 & 21,8 & 82 & 74,5 & 103 & 85,8 & 14 & 12,7 & 3 & 21,4 \\
\hline $1-2 \mathrm{SM}$ & 182 & 40,7 & 2 & 22,2 & 256 & 60,7 & 27 & 24,5 & 16 & 13,3 & 43 & 39,1 & 1 & 7,1 \\
\hline$>2-3 \mathrm{SM}$ & 56 & 12,5 & 2 & 22,2 & 37 & 8,8 & 1 & 0,9 & 1 & 0,8 & 18 & 16,4 & 4 & 28,6 \\
\hline Mais que $3 \mathrm{SM}$ & 30 & 6,7 & 3 & 33,3 & 37 & 8,8 & - & - & - & - & 35 & 31,8 & 6 & 42,9 \\
\hline
\end{tabular}

* Salário Mínimo (SM) na época $=\mathrm{R} \$ 380,00$ 


\section{Discussão}

\section{Limites do estudo}

Dentre os principais limites do presente estudo, ressalta-se o seu desenho transversal, o qual não permite estabelecer relação temporal entre causa e efeito, uma vez que as observações (de exposição e doença) são feitas num mesmo instante. Outro aspecto importante é a possibilidade de ocorrência do viés de prevalência ou sobrevivência, pois apenas os casos existentes são com-

Tabela 3. Prevalência (\%), razões de prevalência e seus respectivos intervalos, de Transtornos do comportamento alimentar (TCAP e BN) entre trabalhadores da zona urbana segundo características socidemográficas, hábitos de vida, obesidade (autorreferida), insatisfação com peso/aparência, problema financeiro/preocupações e agressão na infância. Feira de Santana, BA, 2007.

\begin{tabular}{|c|c|c|c|c|c|c|c|c|}
\hline \multirow{3}{*}{ Variáveis } & \multicolumn{8}{|c|}{ TA } \\
\hline & \multicolumn{4}{|c|}{ TCAP } & \multicolumn{4}{|c|}{$\mathrm{BN}$} \\
\hline & $\begin{array}{c}\text { Prev } \\
(\%)\end{array}$ & $\mathbf{R P}$ & IC & $\begin{array}{l}\text { Valor } \\
\text { de p }\end{array}$ & $\begin{array}{l}\text { Prev } \\
(\%)\end{array}$ & RP & IC & $\begin{array}{l}\text { Valor } \\
\text { de p }\end{array}$ \\
\hline \multicolumn{9}{|l|}{ Sexo } \\
\hline Feminino & 4,3 & 1,04 & $0,61-1,78$ & 0,884 & 1,0 & 1,06 & $0,35-3,22$ & 0,919 \\
\hline Masculino & 4,2 & $*$ & & & 1,0 & $*$ & & \\
\hline \multicolumn{9}{|l|}{ Faixa Etária } \\
\hline Até 35 anos & 5,8 & 2,22 & $1,25-3,93$ & 0,005 & 1,5 & 3,12 & $0,86-11,27$ & $0,093^{1}$ \\
\hline Mais que 35 anos & 2,6 & * & & & 0,5 & $*$ & & \\
\hline \multicolumn{9}{|l|}{ Escolaridade } \\
\hline Analfab./ fundam. & 4,3 & 1,01 & $0,60-1,71$ & 0,965 & 1,3 & 1,48 & $0,50-4,38$ & 0,475 \\
\hline Ensino médio/sup. & 4,2 & $*$ & & & 0,8 & * & & \\
\hline \multicolumn{9}{|l|}{ Cor da pele } \\
\hline Branca/outras ${ }^{3}$ & 4,2 & 0,93 & $0,55-1,59$ & 0,798 & 1,4 & 2,38 & $0,66-8,60$ & $0,258^{1}$ \\
\hline Parda/preta & 4,5 & * & & & 0,6 & * & & \\
\hline \multicolumn{9}{|l|}{ Situação Conjugal } \\
\hline Casado/união estáv & 3,7 & 1,88 & $0,58-6,16$ & $0,454^{1}$ & 1,3 & * & & \\
\hline Div./sep./desq/ viú & 2,0 & * & & & - & - & - & - \\
\hline Solteiro & 5,9 & 2,98 & $0,92-9,71$ & $0,077^{1}$ & 0,9 & 0,68 & $0,21-2,19$ & $1,00^{1}$ \\
\hline \multicolumn{9}{|l|}{ Renda $^{2}$} \\
\hline Até 2 SM & 4,4 & 1,14 & $0,57-2,30$ & 0,712 & 1,1 & 1,27 & $0,28-5,70$ & $1,00^{1}$ \\
\hline Mais que $2 \mathrm{SM}$ & 3,8 & * & & & 0,8 & * & & \\
\hline \multicolumn{9}{|l|}{ CAGE } \\
\hline Sim & 11,0 & 2,94 & $1,53-5,66$ & 0,001 & 3,3 & 3,90 & $1,09-13,91$ & $0,059^{1}$ \\
\hline Não & 3,7 & * & & & 0,8 & * & & \\
\hline \multicolumn{9}{|l|}{ Hábito de fumar } \\
\hline $\operatorname{Sim}$ & 2,4 & 0,52 & $0,14-1,43$ & $0,302^{1}$ & 0,6 & 0,54 & $0,07-4,15$ & $1,00^{1}$ \\
\hline Não & 4,5 & $*$ & & & 1,1 & $*$ & & \\
\hline \multicolumn{9}{|l|}{ Atividade de lazer } \\
\hline Sim & 4,3 & * & & & 1,0 & * & & \\
\hline Não & 4,0 & 0,93 & $0,41-2,14$ & 0,867 & 1,3 & 1,36 & $0,30-6,07$ & $0,659^{1}$ \\
\hline \multicolumn{9}{|c|}{ Insatisfação com peso ou aparência } \\
\hline Sim & 8,3 & 4,22 & $2,35-7,59$ & 0,000 & 2,2 & 6,19 & $1,70-22,62$ & $0,002^{1}$ \\
\hline Não & 2,1 & * & & & 0,4 & * & & \\
\hline \multicolumn{9}{|l|}{ Obesidade (auto-referida) } \\
\hline Sim & 13,5 & 4,49 & $2,35-8,58$ & 0,000 & 1,9 & 2,06 & $0,45-9,43$ & $0,340^{1}$ \\
\hline Não & 3,3 & * & & & 0,9 & * & & \\
\hline \multicolumn{9}{|c|}{ Sofreu agressão na infância } \\
\hline Sim & 9,3 & 2,79 & $1,5-5,19$ & 0,001 & 1,9 & 2,07 & $0,56-7,62$ & $0,262^{1}$ \\
\hline Não & 3,5 & * & & & 0,9 & $*$ & & \\
\hline \multicolumn{9}{|c|}{ Prob. financeiro ou preocupações } \\
\hline Sim & 4,7 & 1,33 & $0,73-2,42$ & 0,345 & 1,4 & 3,07 & $0,68-13,90$ & $0,126^{1}$ \\
\hline Não & 3,6 & * & & & 0,4 & * & & \\
\hline
\end{tabular}

${ }^{1}$ Teste Exato de Fisher $\mid{ }^{2}$ Salário mínimo na época: $\mathrm{R} \$ 380,00 \mid{ }^{3}$ Amarela/indígena $\mid{ }^{*}$ Grupo de referência 
putados, não incluindo óbitos ou curas ${ }^{16}$. Sendo os TA doenças que podem tanto ter duração transitória quanto crônica ${ }^{17}$, este viés não pode ser completamente afastado.

Aspectos metodológicos (população e desenho de estudo, instrumento de coleta de dados e critérios diagnósticos) podem influenciar nos resultados dos estudos sobre TA, seja subestimando ou superestimando as taxas de prevalência ou incidência ${ }^{1,8}$. Nesse sentido, cabe ressaltar que o estudo que originou o banco aqui analisado não foi desenhado especificamente para investigar os TA. Sendo assim, o instrumento de mensuração utilizado não permitiu analisar mais detalhadamente as patologias de interesse, o que pode representar uma série de limitações à análise dos resultados obtidos. Além disso, o presente estudo não se propôs especificamente a diagnosticar casos de TA, o que exigiria também uma avaliação clínica. Dessa forma, embora com um número grande de trabalhadores $(\mathrm{N}=1.273)$, não foi possível realizar inferências estatísticas (o que necessitaria de uma amostra maior da população de trabalhadores de Feira de Santana). Assim, os resultados obtidos se limitam à população estudada.

É possível que a ausência de associações estatisticamente significantes esteja relacionada com o fato de a amostra ter sido pequena em relação ao evento estudado. Entretanto, por se tratar de

Tabela 4. Prevalência (\%), razões de prevalência e seus respectivos intervalos, de Transtornos do comportamento alimentar (TCAP e BN) entre trabalhadores da zona urbana segundo características ocupacionais. Feira de Santana, BA, 2007.

\begin{tabular}{|c|c|c|c|c|c|c|c|c|}
\hline \multirow{3}{*}{ Variáveis } & \multicolumn{8}{|c|}{ TA } \\
\hline & \multicolumn{4}{|c|}{ TCAP } & \multicolumn{4}{|c|}{$\mathrm{BN}$} \\
\hline & $\%$ & RP & IC & Valor de $p$ & $\%$ & $\mathrm{RP}$ & IC & Valor de $p$ \\
\hline \multicolumn{9}{|l|}{ Tipo de vínculo } \\
\hline Formal & 3,8 & * & & & 1,3 & 4,89 & & \\
\hline Informal & 4,3 & 1,11 & $0,61-2,02$ & 0,741 & 0,3 & $*$ & $0,64-37,5$ & $0,124^{1}$ \\
\hline \multicolumn{9}{|l|}{ Ocupação principal } \\
\hline Biscateiro & 5,5 & 1,77 & $0,69-4,54$ & 0,236 & 1,8 & 7,67 & & \\
\hline Autônomo & 4,9 & 1,60 & $0,82-3,14$ & 0,166 & 1,8 & 7,55 & $0,70-83,85$ & $0,11^{1}$ \\
\hline Profissional liberal & - & - & - & - & - & - & $0,95-60,13$ & $0,039^{1}$ \\
\hline Assalariado & 3,1 & * & & & 0,2 & * & - & - \\
\hline Emp. Doméstico & 3,3 & 1,08 & $0,36-3,25$ & $1,00^{1}$ & 1,7 & 7,03 & & \\
\hline Func. Público & 4,5 & 1,47 & $0,54-4,04$ & 0,452 & - & - & $0,64-76,91$ & $0,125^{1}$ \\
\hline Empregador & 7,1 & 2,31 & $0,32-16,47$ & $0,372^{1}$ & - & - & - & - \\
\hline Carga horária & & & & & & & - & - \\
\hline Até 30h & 5,4 & 1,43 & $0,83-2,48$ & 0,196 & 2,3 & 4,12 & & \\
\hline Mais que $30 \mathrm{~h}$ & 3,8 & $*$ & & & 0,6 & $*$ & $1,36-12,51$ & 0,007 \\
\hline
\end{tabular}

${ }^{1}$ Teste Exato de Fisher $\mid{ }^{*}$ Grupo de referência.

Tabela 5. Prevalência (\%), razões de prevalência e seus respectivos intervalos, de Transtornos do comportamento alimentar (TCAP e BN) entre trabalhadores da zona urbana segundo aspectos psicossociais do trabalho. Feira de Santana, BA, 2007.

\begin{tabular}{|c|c|c|c|c|c|c|c|c|}
\hline \multirow{3}{*}{ Variáveis } & \multicolumn{8}{|c|}{ TA } \\
\hline & \multicolumn{4}{|c|}{ TCAP } & \multicolumn{4}{|c|}{$\mathrm{BN}$} \\
\hline & $\%$ & RP & IC & Valor de $p$ & $\%$ & $\mathbf{R P}$ & IC & Valor de $\mathrm{p}$ \\
\hline Baixa exigência & 2,8 & * & & & 0,6 & * & & \\
\hline Trabalho ativo & 4,0 & 1,42 & $0,60-3,35$ & 0,42 & 0,8 & 1,42 & $0,20-9,99$ & $1,00^{1}$ \\
\hline Trabalho passivo & 3,8 & 1,34 & $0,59-3,07$ & 0,48 & 0,9 & 1,68 & $0,28-9,99$ & $0,67^{1}$ \\
\hline Alta exigência & 5,9 & 2,10 & $0,98-4,47$ & 0,05 & 1,7 & 4,15 & $0,81-21,23$ & $0,10^{1}$ \\
\hline
\end{tabular}

${ }^{1}$ Teste Exato de Fisher $\mid{ }^{*}$ Grupo de referência. 
um estudo epidemiológico de base populacional, considera-se que seu caráter exploratório e descritivo é pertinente e relevante. Além disso, fornece oportunidade para estudar os TA em indivíduos não expostos aos vieses inerentes aos estudos realizados com populações clínicas ${ }^{18}$.

Outra limitação encontrada foi a lacuna existente na literatura sobre os TA em trabalhadores em geral, o que limitou a obtenção de parâmetros de comparação com população semelhante à estudada.

\section{Prevalência dos transtornos do comportamento alimentar}

Entre os trabalhadores que apresentaram TA, predominaram as mulheres (aproximadamente $61,3 \%$ dos casos). A diferença entre os sexos não se mostrou tão acentuada como preconiza a literatura (segundo a qual, 90\% dos casos são entre mulheres $)^{4}$. Considera-se a mulher mais vulnerável ao ideal cultural da magreza, o que é considerado um dos fatores predisponentes a esses transtornos ${ }^{17}$.

A prevalência de TCAP entre os trabalhadores $(4,3 \%)$ foi mais elevada do que a prevista na população geral $(2 \text { a } 3 \%)^{5}$. Observou-se similaridade na prevalência entre homens e mulheres: $4,2 \%$ e $4,3 \%$, respectivamente. Frequência similar de TCAP entre homens e mulheres também foi observado por $\mathrm{Hsu}^{19}$.

Elevada prevalência de TCAP tem sido encontrada em outros estudos, a exemplo do estudo de Johnson et al. ${ }^{20}$, com 4.651 mulheres atendidas na atenção básica e em clínicas de ginecologia e obstetrícia nos EUA, utilizando o PHQ, que detectou prevalência de $5,3 \%$.

Diferentemente do referido pela literatura, observou-se prevalência similar de BN entre os sexos $(1,1 \%)$. Embora a prevalência de BN entre os homens ainda não tenha sido totalmente definida, refere-se uma proporção na relação ho$\mathrm{mem} / \mathrm{mulher}$ de $1: 10^{21}$. Estudo realizado com 1.425 oficiais da marinha do sexo masculino também revelou elevada prevalência de BN (6,8\%). Algumas especificidades dessa profissão parecem interferir em tais achados: regulamentos, restrições e estilo de vida regrado ${ }^{21}$. No presente estudo, a prevalência de BN para o sexo masculino foi consideravelmente maior do que o esperado, sugerindo tratar-se de um evento de maior magnitude na população masculina estudada.

Semelhante à maioria dos estudos existentes, os TA foram mais prevalentes entre os mais jovens $^{3}$. As prevalências de TCAP e BN foram dis- cretamente maiores entre aqueles que tinham baixa escolaridade. Estudo realizado por Nunes et al. ${ }^{22}$ também não observaram diferença significativa quanto ao nível escolar e TA. Considerase a escolaridade um fator protetor por ampliar as possibilidades de escolhas na vida, realizações pessoais, autoestima e melhores condições de $\operatorname{vida}^{23}$.

As prevalências de TCAP e BN foram discretamente mais elevadas entre aqueles que tinham baixa renda, diferindo de estudos que consideram os TA mais frequentes entre indivíduos financeiramente favorecidos ${ }^{1}$. A ocorrência de TA segundo aspectos socioeconômicos revela que estes não se restringem a uma classe específica. Embora sejam classicamente associados às classes mais altas da sociedade, recentemente vários autores têm questionado essa relação e demonstrado que ela não é tão consistente quanto parecia ser $^{2}$. Restrição monetária implica em limitações, insegurança e estresse, ou seja, condições onde o bem-estar físico e mental pode ser afetado.

Neste estudo, a distribuição dos TA segundo características sociodemográficas, apontou um perfil de portadores dos transtornos bastante heterogêneo. Borges et al. ${ }^{3}$ ressaltam que, geralmente, o perfil dos portadores de TA é adolescente do sexo feminino, raça branca e alto nível socioeconômico cultural. Contudo, observa-se que esse grupo tem-se modificado: adolescentes do sexo masculino, raça negra e pessoas com nível socioeconômico e cultural baixo têm sido diagnosticadas em relação aos TA.

Maior prevalência de TA entre trabalhadores que apresentaram CAGE positivo estão em consonância com a literatura, a qual aponta relação existente entre abuso de álcool e $\mathrm{TA}^{24,25}$.

As prevalências de TCAP e BN foram significativamente maiores entre os insatisfeitos com o peso/aparência, especialmente em relação à $\mathrm{BN}$. Pessoas insatisfeitas com o peso costumam fazer dieta para emagrecer, o que é considerado um dos fatores precipitantes dos $\mathrm{TA}^{17,26}$. A percepção do peso corporal tem um papel importante na determinação dos comportamentos alimentares anormais, os quais representam fator de risco para o desenvolvimento de $\mathrm{TA}^{20}$.

O TCAP pode estar presente em indivíduos com peso normal ou com obesidade, entretanto é mais comum entre pessoas com excesso de peso ${ }^{19}$. Embora a referência utilizada para a obesidade tenha sido a autopercepção do indivíduo, consideram-se os dados confiáveis, uma vez que estudos epidemiológicos permitem afirmar que o peso e a altura referida pelo indivíduo possuem boa 
correlação com os dados aferidos ${ }^{27}$. Sabe-se que mais do que o índice de massa corporal, o sentirse gordo, tem mais influência sobre comportamentos alimentares inadequados e utilização de métodos inapropriados para perda de peso ${ }^{22}$.

Os trabalhadores com relato de agressão na infância apresentaram maiores prevalências de TCAP e BN do que aqueles que não vivenciaram este tipo de experiência. A agressão física ou sexual é responsável por vários problemas psíquicos e também pode ter relação com os $\mathrm{TA}^{28}$. Deve-se considerar ainda que, por se tratar de um tema delicado, é possível ter havido omissão de informações e, consequentemente, subestimação dos dados.

Os trabalhadores informais tiveram maior prevalência de TCAP e BN. Empregadores/empresários, biscateiros, autônomos e funcionários públicos, em ordem decrescente, foram as ocupações com maiores prevalências de TCAP. Os biscateiros, autônomos e os empregados domésticos apresentaram maiores prevalências de BN.

A lacuna existente na literatura impossibilitou estabelecer comparação entre os achados referentes aos aspectos do trabalho deste estudo (vínculo, ocupação e carga horária semanal) com outras pesquisas similares, pois as pesquisas realizadas com grupos ocupacionais de risco para TA (atletas, modelos, dançarinos) limitam-se às questões relacionadas à imagem ou ao peso corporal. Contudo, estudos sobre os TA realizados com outras populações/amostra permitem fazer algumas inferências, especialmente em relação ao efeito do estresse sobre o comportamento alimentar. Em pesquisa com estudantes de medicina observou-se que a elevada taxa de prevalência de TA encontrada estava relacionada com alto nível de estresse dos estudantes em decorrência de elevada carga horária, atividades curriculares e extracurriculares e autocobrança ${ }^{29}$.

No presente estudo, buscou-se avaliar o estresse ocupacional através do Modelo DemandaControle $^{8}$. Observou-se maior prevalência de BN e TCAP no grupo de trabalho de alta exigência (situação em que a atividade laboral é realizada sob alta demanda psicológica e baixo controle). Esta situação é considerada responsável por efeitos negativos sobre a saúde, incluindo transtornos mentais comuns e doenças cardiovasculares ${ }^{8,13,30,31}$.

Embora sejam escassas as pesquisas que abordam a relação específica entre TA e aspectos psicossociais do trabalho, pode-se afirmar que os dados encontrados são concordantes com os achados de estudos sobre saúde do trabalhador, os quais têm produzido evidências consistentes de que a atividade laboral exercida em condições de baixo controle e alta demanda (alta exigência) representa um fator de exposição para a saúde dos trabalhadores ${ }^{8,13,32}$.

\section{Conclusão}

Este estudo epidemiológico enfocou uma temática ainda pouco estudada (trabalho e transtornos alimentares). Outro diferencial do estudo foi analisar o evento de interesse a partir de dados de uma investigação de base populacional, diferentemente da maioria dos estudos sobre TA que foram realizados em serviços de saúde, o que amplia a abrangência deste estudo.

O perfil obtido dos trabalhadores, segundo as variáveis estudadas, revelou prevalências de TCAP e BN acima das previstas pela literatura. Esses dados devem ser vistos com cautela uma vez que as estimativas de prevalência ou incidência dos TA parecem depender dos métodos de mensuração adotados ${ }^{2}$.

Este estudo buscou dar visibilidade a possíveis relações entre os aspectos relacionados ao trabalho e TA. Os resultados apresentados poderão servir de subsídios para ações voltadas para a saúde do trabalhador. Entretanto, por se tratar de um estudo inicial e de caráter exploratório, faz-se necessária a continuidade de investigações na área, a fim de ampliar e dar consistência ao conhecimento existente.

\section{Colaboradores}

APK Prisco, TM Araújo e MMG Almeida participaram do delineamento da proposta de estudo; da coleta dos dados, da análise e da interpretação dos dados; e da elaboração e revisão do manuscrito. KOB Santos realizou revisão da análise dos dados e do manuscrito. 


\section{Referências}

1. Hsu LKG. Epidemiology of eating disorders. Psychiatry Clin North Am 1996; 19(4):681-700.

2. Nunes MA. Epidemiologia dos transtornos alimentares. In: Nunes MA, Appolinário JC, Galvão AL, Coutinho W, organizadores. Transtornos Alimentares e Obesidade. 2a Edição. Porto Alegre: Artmed; 2006. p. 51-57.

3. Borges NJBG, Sicchieri JMF, Ribeiro RPP, Marchini JS, Santos JE. Transtornos alimentares - Quadro clínico. Medicina, Ribeirão Preto 2006; 39(3):340-348.

4. Bucaretchi HA, Cordás TA. Distúrbios alimentares: anorexia e bulimia. In: Quayle J, Lúcia MCS, organizadores. Adoecer: as interações do doente com a sua doença. São Paulo: Atheneu; 2003. p. 49-60.

5. Cordás TA. Transtornos alimentares em discussão. Rev Bras Psiquiatr 2001; 23(4):178-179.

6. Brito JC, D’Acri V. Referencial de análise para o estudo da relação trabalho, mulher e saúde. Cad Saude Publica 1991; 7(2):210-214.

7. Silva CM. Perturbações alimentares em contextos desportivos: Um estudo comparativo. Análise Psicológica 2001; 19(1):131-141.

8. Karasek RA. Job Demand, Job decision latitude, and mental strain: implications for job redesign. Administrative Science Quarterly 1979; 24:285-308.

9. Rebelo A, Leal I. Factores de personalidade e comportamento alimentar em mulheres portuguesas com obesidade mórbida: Estudo exploratório. Análise Psicológica 2007; 25(3):467-477.

10. Instituto Brasileiro de Geografia e Estatística (IBGE). Indicadores sociais mínimos. Pesquisa nacional por amostras de domicílios. IBGE: Rio de Janeiro; 2007.

11. Organização Mundial de Saúde (OMS). Relatório sobre a saúde no mundo 2001: Saúde Mental: Nova Concepção, Nova esperança. Lisboa: OMS; 2002.

12. Grucza RA, Prybeck TR, Cloninger CR. Prevalence and correlates of binge eating disorder in a community sample. Compr Psychiatry 2007; 48(2):124131.

13. Spitzer LR, Kroenke K, Williams JBW. Validation and utility of a self-report version of PRIME-MD the PHQ Primary Care Study. JAMA 1999; 282(18):1737-1744.

14. Karasek RA. Job Content Questionnaire and User's Guide. Massachusetts: University of Massachusetts; 1985.

15. Araújo TM, Aquino E, Menezes G, Santos CO, Aguiar L. Aspectos psicossociais do trabalho e distúrbios psíquicos entre trabalhadoras de enfermagem. Rev Saude Publica 2003; 37(4):424-433.

16. Pereira MG. Epidemiologia teoria e prática. Rio de Janeiro: Guanabara Koogan; 1995.

17. Morgan CM, Vevvhiatti IR, Negrão AB. Etiologia dos transtornos alimentares: aspectos biológicos, psicológicos e sócio-culturais. Rev Bras Psiquiatr 2002; 24(Supl. III):18-23.

18. Hay PJ. Epidemiologiados transtornos alimentares: estado atual e desenvolvimentos futuros. Rev Bras Psiquiatr 2002; 4(Supl. III):13-17.

19. Hsu LKG. Eating Disorders: Pratical Interventions. JAMWA 2004; 59(2):113-124.
20. Johnson JG, Spitzer RL, Willams JBW. Health prob lems, impairment and illnesses associeated with bulimia nervosa and binge eating disorder among primary care and obstetric gynaecology patients. Psychol Med 2001; 31(8):1455-1466.

21. Mcnulty PAF. Prevalence and Contributing Factores of Eating Disorder Behaviors in Active Duty Navy Men. Military Medicine 1997; 162(11):753-758.

22. Nunes MA, Barros FC, Olinto MTA, Camey S, Mari JDJ. Prevalence of abnormal eating behaviors and inappropriate methodos of weitgh control in young women from Brazil: A population-based study. Eat Weight Disord 2003; 8(2):100-106.

23. Ludermir AB, Melo-Filho DA. Condições de vida e estrutura ocupacional associadas a transtornos mentais comuns. Rev Saude Publica 2002; 36(2):213-221.

24. Jorge MR. Manual diagnóstico e estatístico de transtornos mentais. DSM-IV-TRTM: texto revisado. $4^{\mathrm{a}}$ Edição. Porto Alegre: Artemed; 2002.

25. Arias JE, Hawke JM, Arias AJ, Kaminer Y. Eating disorders symptoms and alcohol use among adolescents in substance abuse treatment. Substance Abuse: Research an Treatment, 2009; 3:81-91. [acessado 2012 fev 13]. Disponível em: http://www.la-press.com/ eating-disorder-symptoms-and-alcohol-use-amongadolescents-in-substanc-article-a1738-related

26. Nunes MA, Olinto MTA, Camey S, Morgan C, Mari J. Abnormal eating behaviors in adolescent and young adult women from southern Brazil: reassessment after four years. Soc Psychiatry Psychiatr Epidemiol 2006; 41(12):951-956.

27. Schmidt MI, Duncan BB, Tavares M, Polanczyk CA, Pellanda L, Zimmer PM. Validity of sef-reported weight: a study of urban brazilian adults. Rev Saude Publica 1993; 27(4):271-276.

28. Nodin N. A depressão nas perturbações da alimentação. Análise Psicológica 1999; 17(4):687-694.

29. Souza FGM, Martins MCR, Monteiro FCC, Menezes-Neto GC, Ribeiro I. Anorexia e bulimia nervosa em alunas da Faculdade de Medicina da Universidade Federal do Ceará - UFC. Revi Psiquiatr Clín 2002; 29(4):172-180.

30. Block JP, He Y, Zaslawsky AM, Ding L, Ayanian JZ. Psychosocial stress change in weight among US adults. Am J Epidemiol 2009; 170(2):181-192.

31. Jacobson IG, Smith TC, Smith B, Keel PK, Amoroso PJ, Wells TS, Bathalon GP, Boyko EJ, Ryan MAK. Disordered eating and weight changes after deployement: longitudinal assessment of a large US military cohort. Am J Epidemiol 2009; 169(4):415-427.

32. Takaki J, Minoura A, Irimajiri A, Hibino Y, Kanbara S, Sakano N, Ogino K. Interactive effects of job stress and body mass index on over-eating. $J$ Ocup Health 2010; 52(1):66-73.

Artigo apresentado em 30/10/2011

Aprovado em 15/02/2012

Versão final apresentada em 29/02/2012 